\title{
BREKEKEKEX KOAX KOAX Grenouilles-déesses de Grèce
}

\author{
PIERRE LÉVÊQUE \\ Centre de Recherches d'Histoire Ancienne \\ Faculté de Lettres \\ Université de Franche-Comté (Besançon)
}

RESUMO: A partir da referência a rãs, na inscrição da base de um trípode déffico, pretende-se, à luz de dados epigráficos e líterários, definir a figura de Deusas-rãs na Grécia. Assim, na Macedônia, existlu uma Ártemis-rã (Ártemis Blaganîtis); o simbolismo religioso das rãs transparece nas Rås de Aristófanes, onde elas constituem o coro. Sua importância é assinalada ao se comparar o texto de Aristófanes com um hino do Rig-Veda $(\mathrm{VII}, 13)$ consagrado às rãs. Pouco integrada no mito, deve-se, no entanto, ressaltar alguns mitos de transformação de Inimigos em rãs, na Grécia e também em outras culturas. Finalmente o estudo do vocabulário acrescenta novas luzes à documentação discutida. A rã não é uma protagonista, como outros animais, no imaginário grego, mas representa também um papel que merece destacar. Tem uma função semântica que é a de significar a vida saltitante e criativa do melo aquático e dos animais que o povoam. Está ligada à mulher, associada à sua vida sexual, encarna a fecundidade, a fertilidade, a renovação do mundo. Dal ser próxima das deusas a quem ela é ofertada e das quais é uma saltitante hipostase, quer se trate de Mães ou de uma filha como Ártemis, que se deleita também na umidade dos pântanos.

PALAVRAS-CHAVE: Rãs, deusas-rãs, literatura, mitologia, religião, Grécia.

Cette base du trépied est énigmatique et, avant de déclencher les curiosités avides des modernes, elle questionnait les Anciens, comme on le voit bien dans deux textes de Plutarque. Dans le Banquet des sept sages (164 A), Pittacos demande a Périandre la raison de ces grenouilles et quelles relations elles ont avec le dieu Apollon ou avec le dédicant. Le tyran refuse de répondre tant qu'il n'aura pas appris ce que veulent dire le Rien de trop et le Connais-toi toi-même.

Dans les Oracles de la Pythie (399 F), Diogenianus s'étonne de ces grenouilles et de ces serpents d'eau: le palmier ne pousse pas dans les marais et n'aime pas l'eau; la grenouille n'a aucune relation avec les Corinthiens et le dieu préfère 
corbeaux, cygnes, loups et faucons. Sérapion donne alors un avis motivé: "Lauteur aurait voulu signifier par là que l'élément liquide fournit au soleil le principe de sa nourriture, de sa naissance et de sa respiration, soit qu'il se souvint du vers d'Homère: Et le soleil levant monta du lac splendide, soit qu'il sût que les Egyptiens prenaient comme symbole de l'origine et du lever de l'astre un enfant nouveau-né assis sur un lotus". La discussion continue à partir de cette interprétation stoïcienne. Et grenouille de reparaître, dans ses connexions avec le soleil (400 B-C): "Vous représentez ce même soleil comme un être vivant issu de la terre ou comme une plante de marécages, en l'inscrivant d'office dans la patrie des grenouilles et des serpents d'eau! Laissons plutôt cela au fatras mélodramatique des Stoïciens et ce que les artistes ont fait d'accessoire, considérons-le aussi comme accessoire." Sage proposition; sinon, la grenouille finirait par devenir aussi grosse que le palmier auquel elle ne fournissait qu'un décor, ce qui n'est pas à dire que ce décor était privé de sens.

Saluons au passage l'interprétation de Waldémar Deonna qui a consacré tant d'articles aux batraciens et un mémoire spécial à ce monument delphique et qui conclut qu'il n'y a rien d'étonnant à ce que le palmier, symbole de fertilité, de fécondité, de durée, de renaissance, de victoire, s'appuie sur ces animaux fécondants et créateurs que sont les hydres et les grenouilles.

Retenons en tout cas que la symbolique de la grenouille pèse déjà lourd chez les Anciens, et que les penseurs qui entourent Plutarque dans cet entretien ne pensent pas pouvoir résoudre le problème sans sortir de Grèce et sans évoquer les antécédents égyptiens.

Si nous rouvrons le dossier des grenouilles, c'est que l'exégèse d'inscriptions macédoniennes connues depuis 1939 , mais jamais vraiment comprises, vient d'être présentée par Miltiade Hatzopoulos (1987, 397 sq.) et qu'elle introduit une Artémis Juste Grenouille. Il s'agit d'une pierre inscrite exhumée à Palatitsa et qui fait partie d'un lot de 14 inscriptions sur un autel voué à une Artémis (parfois simplement théa) dite Digaia Blaganeîtis/Blaganîtis (ou en Blagánois).

S'appuyant sur une glose d'Hesychius blakhán: ho bátrakhos, sur la présence vraisemblable aussi dans un acte d'affranchissement de la région d'Edessa-Béroia (ibidem, 399-380) d'une déesse des grenouilles, Theâi tôn batrákhon, le savant épigraphiste propose de voir dans cette Blaganîtis la déesse des grenouilles.

Certes le premier problème qu'il doit se poser concerne la philologie: le Blakhán d'Hesychius (avec un khi) peut-il être assimilé au Blaganîtis de l'inscription (qui comporte un gamma)? Il est résolu dans une démonstration, appuyée sur nombre d'exemples empruntés au dialecte macédonien, et qui se révèle pleinement convaincante. Nous nous trouvons donc en présence d'une donnée nouvelle: une Artémis (ou déesse) grenouille, tout à fait inconnue jusqu'ici, qui est en outre qualifiée de Digaía, c'est-à-dire, en appliquant les mêmes équations phonétiques, l'homologue de Dikaía, la Juste, dans les autres dialectes grecs.

Cette Blaganîtis ne retient pas moins l'attention de l'historien des religions que celle du philologue. Il faut d'abord liquider le mauvais conseil donné par L. Robert (Hatzopoulos, 1987, 400-401) d'attribuer un sens purement local à cette épiclèse, conseil dont on voit combien il est réducteur de la réalité religieuse. Les grenouilles de l'Haliacmon ne sont pas en cause, ou pas seules en cause; sinon en combien de lieux en Grèce aurions-nous une Artémis Grenouille?

Au reste, l'exégète du texte sait bien qu'en Grèce, la problématique de la grenouille s'étend largement dans le temps et dans l'espace et, bien que ce ne soit pas son propos, il fournit un faisceau d'indices: 
- les statuettes de grenouilles exhumées sur le site voisin de Néa Nikomédeia et qui datent du Néolithique ancien (400);

- les anthroponymes formés sur le nom de la grenouille et qui sont signalés en Grèce septentrionale, en Thessalie notamment (401, n.13b et Addendum, 412);

- les statuettes votives en bronze du sanctuaire d'Artémis Orthia à Sparte (Addendum, 412).

Au-delà de ces notations auxquelles se limite volontairement Miltiade Hatzopoulos, il y a tout un dossier concernant l'imaginaire de la grenouille en Grèce que nous souhaitons rouvrir, sans avoir la prétention d'être exhaustif.

Nous exposerons ce que la littérature, les mythes, le vocabulaire peuvent nous apprendre.

$\mathrm{Si}$ nous commençons par la littérature, il faut d'abord exclure de notre enquête deux textes qui ne nous apportent pas grand-chose: la Batrakhomyomakhie (Combat des rats et des grenouilles) et les fables.

La Batrakhomyomakhie était attribuée à Homère. C'est, en fait, un pastiche des combats de l'Iliade, mettant en scène d'humbles animaux autour, non d'une ville assiégée, mais d'un marécage. Naturellement les dieux sont présents. Sur la demande de Zeus, Athéna répond qu'elle ne soutiendra aucun des adversaires, car les rats mangent ses vêtements et les grenouilles l'empêchent de dormir de leurs cris. Cependant le père des dieux a pitié des pauvres grenouilles, en train d'être anéanties: il accepte de lancer sa foudre, comme Arès l'en prie, et il envoie des secours aux batraciens en la personne des crabes qui rongent à pleine bouche la queue des rats. La parodie du grandiose épique est d'autant plus forte et plus plaisante qu'elle met en scène des animaux pour lesquels il est difficile de se passionner.

Quant aux fables, voici un relevé des thèmes principaux. Lenquête est faite à partir de la totalité du corpus antique (Perny, 1965): Esope (E), tradition ésopique (TE), Phèdre (P. époque d'Auguste) et Babrius B (seconde moitié du $\mathrm{I}^{\text {er }}$ siècle après JésusChrist). Rien de positif dans le tableau qui en résulte: les grenouilles sont faibles et craintives, ce qui ne les empêche pas d'être vantardes et méchantes, quand elles trouvent moins fort qu'elles. Peinture traditionnelle et psychologie convenue d'un animal qui se sauve dès qu'on l'approche et à qui l'on ne reconnaît ni courage ni vertu.

vanité et vantardise

aussi grosses que boeuf et vache

faux exercice de la médecine

P24 B27

E69 B120

méchanceté sournoise noyade de la souris

TE384

incapacité à s'organiser

demandent un roi

E66 P2

faiblesse

impuissantes à aider

TE90

encore plus faibles que les lièvres $\quad$ E191

inconscientes du danger

E67 TE69

craintes constantes

craignent l'asséchement du puits

craignent le mariage du soleil

E68 TE43 et 63

craignent les combats de taureaux mâles

P6 $\quad$ B24 
Si les grenouilles de la fable sont par trop insignifiantes, il est une fameuse comédie d'Aristophane dont elles sont les choreutes-titres. Les batraciens ne sont pas ici seulement destinés à provoquer Dionysos déguisé en Héraclès pour sa descente aux infernaux palus; ils révèlent leur nature profonde de démons des eaux. Ces filles des ondes, "enfants lacustres des fontaines" (vers 210) - comme il est dit ailleurs des fleuves (Motte, 162) -, se caractérisent elles-mêmes par l'harmonie de leurs chants et par leurs liens étroits avec de jeunes dieux. Alors qu'elles chantent et dansent leur limpidité aquatique, Dionysos se montre le plus ignoble des personnages, tonitruant et pétant de toutes ses forces, n'ayant à la bouche que son croupion (órrys) ou son anus (proktós) en sueur. Elles, sont entourées d'une aura de musique et d'harmonie. "Elles se réjouissent de leurs mélodies entrecoupées de plongeons" (vers 244-246). Elles créent un mot immense de 19 lettres pour évoquer le pétillement des bulles (247): pompholygopaphlásmasi. Les gutturales, si nombreuses dans leur chant de triomphe, s'effacent dans l'harmonie et elles créent une poésie qui, comme souvent, est la forme la plus sublime de la troisième fonction. Elles ne sont que musique délicate, dans toutes les circonstances: "Aux jours de soleil, nous sautâmes parmi le souchet et le jonc, joyeuses de nos mélodies entrecoupées de mille plongeons; ou, fuyant l'averse de Zeus, au fond de l'eau, nous entonnâmes d'allègres choeurs de danse au bruissement des bulles" (vers 242 sq.). Leurs jeux d'eau dans la gloire créative des marais, fussent-ils infernaux, donnent à leur choeur assez de personnalité religieuse pour préparer le vrai choeur, celui des initiés. On est dans un monde à part, ce dont témoigne aussi le schéma métrique, si étrange, si difficile à définir (so singular a composition: White, 159), fondé surtout sur des dimètres trochaïques et iambiques et sur quelques dactyles lyriques.

On pourrait chicaner sur les dieux invoqués comme amis par les grenouilles. L. Bodson $(1978,60)$ estime que "le contexte dans lequel Aristophane insère la déclaration solennelle n'est pas sans importance: c'est pour défendre leur chant raillé par Dionysos que les grenouilles invoquent, en tant que divinités de la musique, Apollon..., les Muses et Pan".

Cela est vrai. Les dieux à qui en appellent nos batraciens sont des professionnels de la musique et c'est en tant que tels que leur sympathie pour les grenouilles est rappelée. Mais, chose étonnante, Dionysos - qui se conduit envers elles de manière si ignoble et qui fait honte aux dieux en face de ces animaux inspirés - est prié le premier, bien qu'il ne soit pas spécialement une divinité musicale, et ce dans une atmosphère cultuelle bien définie, au Limnaion, dans la journée en partie funeste des Marmites saintes, "quand la foule des hommes s'avance en [leur] pourpris": vers 219 , où il faut bien noter que le quartier des Marais, dont on connaît les cultes chthoniens et où sont localisées les plus anciennes fêtes de Dionysos (Thucydide, 2,15 ), est qualifié de sanctuaire des grenouilles (téménos): khorê̂ kal emon témenos laôn ókhlos.

Le choeur des Grenouilles est un document d'une exceptionnelle importance pour la connaissance de ces démons des eaux, si gais, si chaleureux dans leur participation à la vie grouillante des marais et à sa reproduction, et qui incarnent si fortement les puissances de l'élan vital, avec un intérêt pour les morts, comme on le voit bien dans la fête des Marmites dont les résonances funéraires sont claires.

Ce texte prende d'autant plus d'importance qu'on peut le comparer à un hymme du Rig-Véda (VII, 13) qui est voué aux grenouilles: beaucoup plus religieux, mystique 
même, célébrant avec ces animaux le premier jour de la mousson, solennel entre tous dans la mesure où il ranime, en présence des brahmanes, la vie du cosmos dans une liturgie de l'éternel retour, le poème indien participe de la même ferveur, du même enthousiasme envers la gent des marais que le choeur d'Aristophane.

Au surplus, ces savantes compositions littéraires reposent sur des formes populaires, non littérarisées, antérieures. Jusque dans le folklore français (Sébillot, réimpression 1968, III, 260) subsistent des saynètes inspirées par les croassements des grenouilles et où s'engage un authentique dialogue entre un coryphée et une sorte de choeur.

La grenouille est peu intégrée dans le mythe. On s'en rend compte, si l'on constate que le mot de bátrakhos ne figure pas une seule fois dans la Bibliothèque d'Apollodore. Ce qui est signifiant, car ce mythographe du $2^{e}$ ou $3^{e}$ siècle de notre ère fait la synthèse de tous les grands mythes. Il ne sert de rien non plus de tenter des rapprochements non convaincants, comme on a voulu le faire en transformant le héros Brotéas (fils de Tantale) en héros-grenouille, en ne se fondant que sur la (très vague!) homologie des noms (Eitrem, 1982, 113-113).

Restent seulement des mythes de transformation d'ennemis en grenouilles. Notons d'abord que, de tous les phénomènes merveilleux qu'on rencontre dans les récits populaires, la métamorphose est "sans doute l'opération magique la plus fréquente et la plus spectaculaire" (Jean, 1981, p.66). Ces métamorphoses, tout en mettant la gent grénouille en connexion avec l'eau et dans l'étroite mouvance d'une déesse Mère (au moins dans le premier d'entre eux), positionnent cependant la grenouille à sa place dans l'échelle des êtres (inférieure à celle de l'homme).

Une légende importante, localisée en Lycie, berceau des Létoïdes, met la grenouille en connexion étroite avec Léto, mère féconde d'Artémis et d'Apollon. Les bouviers de Xanthos avaient tenté d'empêcher la déesse d'approcher d'un étang où elle voulait s'abreuver avec ses enfants (Antoninus Liberalis, 35; Ovide, Métamorphoses, 6, 317-381; cf. Gallet de Santerre, 1958, 145; Hatzopoulos, 1987, 399, n.9a). La transformation est narrée dans Ovide en termes particulièrement sinistres. À preuve la fin du récit $(6,380-381)$ :

Leur tête rejoint leurs épaules et leur cou disparât'; leur échine verdit; leur ventre, la plus grande partie de leur corps, blanchissent. De nouveaux êtres sautent dans les profondeurs bourbeuses, des grenouilles.

Cet épisode a été justement comparé à un mythe australien, de fait très proche (Lang, réimpression 1968, 147-148; Deonna, 1951, 20):

Une femme met au monde deux enfants et cherche une source pour les y baigner; mais des pâtres l'éloignent d'une fontaine qui, disent-ils, sert à abreuver leurs bestiaux; des loups la conduisent d̀ une rivière où elle peut se désaltérer et baigner ses enfants. De retour à la fontaine, elle change en grenouilles les pâtres qui s'y baignent et c'est depuis que les grenouilles vivent dans des marais et au bord des rivières.

Mythe qui est plus complexe que le grec, puisqu'il est étiologique et sert donc à donner une explication à la localisation des batraciens dans des lieux humides.

On peut aussi penser à un mythe particulièrement rare, celui d'Euphorion (Ptolémée Héphaistion, Novae historiae libri 4, 1): Euphorion l'ailé, né des amours d'Achille et d'Hélène, est poursuivi par Zeus, à qui il résiste en fuyant. Rejoint à 
Mélos, il est tué d'un coup de foudre. Les Nymphes l'enterrent, mais Zeus, dans sa colère, les change en grenouilles.

Ces transformations en grenouille comme punition ne sont pas rares dans la mythopoiétique universelle. Je veux en donner un exemple, mésopotamien, concernant Inanna, la Mère suprême du panthéon sumérien, qu'on trouve dans une version assyrienne, donc tardive, de l'Épopée de Gilgamesh. La déesse est présentée par le héros comme une allumeuse:

Tu as aimé le jardinier de ton père qui ne cessait de t'offrir des dattes par couffins et te procurait tous les jours un menu plantureux. Tu avais levé les yeux sur lui et tu l'étais allée provoquer: Jouissons de ta vigueur, ô mon Ishullanu. Oh! sors la 'main' et me touche la vulve... Et puis tu l'as frappé et changé en grenouille assignée en résidence dans ton palais [Bottéro, dans Kramer, 1983, 177].

Cette version ninivite tardive fait d'Inanna une effrontée passant de bras en bras: c'est méconnaître sa nature essentielle de Grande Mère de fécondité dont les étreintes renouvellent l'univers. L'épisode est, au reste, intéressant, parce qu'il narre ses amours avec un jardinier: le jeu, très librement évoqué ici, des organes sexuels introduit dans un monde d'abondance rurale, fruit de l'hiérogamie qui unit la déesse suprême avec son amant-horticulteur.

Et l'on peut bien se demander quel est le sens profond et réel de la métamorphose en grenouille, adjuvant de la reine des dieux et vivant à jamais en son palais? N'est-ce pas une magie d'héroïsation d'un humble jardinier qui a su exciter les ardeurs sexuelles de l'insatiable déité?

Le recours au folklore n'est pas sans intérêt. Retenons ici deux contes du folklore français (Sébillot, réimpression 1968, II 177 et 411). Un couple de jeunes amoureux trouve enfin la sérénité, transformé en grenouilles d'or cachées dans une fontaine (origine: Bretagne). Une méchante fée change en grenouille son amoureux, qu'elle porte ensuite au vivier. La transformation est ambivalente et je note que, dans ce dernier cas, c'est l'action d'une fée = déesse qui liquide (ou glorifie) son amant. Ces mythes mettent en scène un personnel divin féminin.

Létude du vocabulaire peut encore ajouter à notre documentation. Il y a deux mots en grec pour désigner la grenouille et le crapaud, le plus souvent mal distingués. Bátrakhos est la grenouille. P. Chantraine (s.v.) confirme que le mot n'a pas d'étymologie établie. Il pose le problème d'un rapport avec le latin bothrax, lézard et se demande, du fait de la multiplicité des formes du mot s'il n'y a pas ici "l'action d'un tabou linguistique". Le cas de phryné, plutôt le crapaud, est très différent: le mot est indo-européen, mais la racine désigne seulement un animal de couleur marron et qui, en celtique et en germanique, s'applique non aux batraciens, mais aux castors (Chantraine, s.v., qui rappelle que les batraciens n'ont pas de nom en indo-européen commun).

Les anthroponymes crés sur ces deux noms ne sont pas rares. Pour bátrakhos Hatzopoulos (1987, 412, Addendum) en signale de nouveaux en Thessalie. Ceux qui sont formés sur phryné sont encore beaucoup plus nombreux: P. Chantraine en dresse une liste considérable, en remarquant que certains exemples remontent au $7^{\mathrm{e}}$ siècle: Phrynion, Phrynon, Phrynichos, Phryniskos, Phrinitas, et au féminin, Phryné et Phrynis. Tous ces mots sont de bon augure et, si la fameuse courtisane Phryné (qu'elle soit une ou plusieurs, peu nous importe ici) a emprunté ce nom, ce n'est évidemment pas que cette très belle fille eût la bouche trop grande et le 
nez camus (hypothèse examinée sérieusement par A. Raubitschek, PW, s.v., 1941), mais parce que c'était une brune ravissante...

On n'a aucune raison de supposer non plus que la vieille Phryné, aux épouvantables manières, qu'Aristophane appelle aussi du nom de la larve Empousa (Assemblée des femmes, 1101 et 1056) montre un dégoût du crapaud. Elle était une jolie brunette, mais il y a fort longtemps et c'est de cette ambigüité que vient le comique du passage.

Sans être jamais, dans l'imaginaire grec, une protagoniste - comme le sont l'ours et le loup, deux grands prédateurs qu'il fallait se propitier -, la grenouille y joue sa partie. Elle a une fonction sémantique qui est de signifier la vie bondissante et créatice du milieu aquatique et des animaux qui le peuplent. Elle est liée à la femme, associée à sa vie sexuelle, "symbole de la matière primitive, humide, informe" (Deonna 1951, 28 et surtout 1952). Elle incarne la fécondité, la fertilité, le renouvellement du monde. Sa morphologie fait d'elle une femme ouverte, directement en prise sur le renouveau de l'univers. Il n'est pas étonnant qu'elle soit si proche des déesses à qui on l'offre et dont elle est une bondissante hypostase, qu'il s'agisse de Mères ou d'une fille comme Artémis, qui se complaît elle aussi dans l'humidité des marais.

Déjà plusieurs niveaux de compréhension sont apparus. Nous pourrons peutêtre en savoir davantage en menant une enquête plus poussée dans l'espace et dans le temps et en évoquant aussi les documents figurés. Lintégration de la grenouille dans les cultes en Macédoine depuis le Néolithique jusqu'à l'époque romaine laisse entendre que cette tentative n'est pas vaine...

\section{Notes}

1- Aristophane dit lui-même (Grenouilles, 1060) "qu'il sied que les demi-dieux usent de termes plus grandioses", ce qui contribue à donner une aura divine à ses grenouilles.

2- Le mot bátrakhos est toujours masculin; mais à côté de phryné, féminin, le plus courant, il y a un phroanos au masculin.

3- Ce mot de basse latinité n'a lui-même de rapprochement possible qu'avec botrakhos, doublet de batrakhos (Meillet-Ernout, s. v.). Boisacq proposait un rapprochement qu'il juge mui-même "pas très sûr" avec v. h. a. chrota, crapaud.

4- Ce que n'infirment pas les rapports étroits du latin rana (dérivé peut-être de racco, crier) et du celtique: irl. et britt. ran (Meillet et Ernout, s. v.).

\section{Références Bibliographiques}

BODSON, L. Hiera Zoia. Bruxelles: 1978.

CHANTRAINE, P. Dictionnaire étymologique de la langue grecque. Paris: Klincksieck, $1968 s q$.

DEONNA, W. Sauriens et batraciens. Revue des Études Grecques. Paris, p.32 sq., 1919. DEONNA, W. L'ex-voto de Cypselos à Delphes. Revue de l'Histoire des Religions. Paris, 139 et 140, 1, p.162 sq., et 2, p.5 sq., 1951. 
DEONNA, W. La femme et la grenouille. Gazette des Beaux-Arts. Paris, p.230 sq., 1952.

EITREM, S. Varia, Symboles Osloenses. Oslo: 1932.

GALLET DE SANTERRE, H. Délos primitive et archaïque. Paris: De Boccard, 1958. HATZOPOULOS, M.-B. Artémis Digaia Blaganitis en Macédoine. Bulletin de Correspondance Hellénique. Paris, p.397 sq., 1987.

JEAN, G. Le pouvoir des contes. Paris: 1981.

LANG, A. Myth, ritual and religion. Londres: 1906, réimpression New York: 1968. MOTTE, A. Prairies et jardins de la Grèce antique. Bruxelles: Palais des Académies, 1973.

PERNY, B.-E. Babrius and Phaedrus. Harvard: 1965.

SEBILLOT, P. Le folk-lore de France (réimpression). Paris: E. Guilmoto, 1968.

WHITE, J.-W. The verse of Greek comedy. Londres: 1912.

LEVÊQUE, P. BREKEKEKEX KOAX KOAX. Grenouilles - déesses de Gréce. Classica. São Paulo, 5/6: 149-156, 1992/1993.

RESUME: A partir de la référence aux grenouilles dans une inscription de la base d'un trépied delphique, on prétend, à la lumiére de données épigraphiques et littéraires, définir la figure de Déesses-grenouilles en Grèce, Ainsi, en Macédoine, il y a eu une Artémis-grenouille (Artémis Blaganîtis); le symbolisme religieux des grenouilles apparaît dans les Grenouilles d'Aristophane, où elles constituent le choeur. Son importance est signalée en comparant le texte d' Aristophane avec un hymme du Rig-Véda (VII, 13) consacré aux grenouilles. Peu integrées au mythe, on doit, néanmoins, souligner quelques mythes de transformations d'ennemis en grenouilles, soit en Grèce soit également dans d'autres cultures. Finalemente, l'étude du vocabulaire ajoute une lumière nouvelle à la documentation discutée. La grenouille n'est pas une protagoniste, comme le sont d'autres animaux, dans l'imaginaire grec, mais y joue aussi sa partie. Elle a une fonction sémantique qui est de signifier la vie bondissante et créatice du milieu aquatique et des animaux qui le peuplent. Elle est liée à la femme, associée à sa vie sexuelle, elle incarne la fécondité, la fertilité, le renouvellement du monde. II n'est pas étonnant qu'elle soit si proche des déesses à qui on l'offre et dont elle est une bondissante hypostase, qu'il s'agisse de Mères ou d'une fille comme Artémis, qui se complaît elle aussi dans l'humidité des marais.

MOTS CLÉS: Grenouilles, déesses-grenouilles, littérature, mythologie, religion, Grèce. 